\title{
A Comparison of CT Contrast Enhancement and BUDR Labeling Indices in Moderately and Highly Anaplastic Astrocytomas of the Cerebral Hemispheres
}

\author{
Michael W. McDermott, Hendrikus G.J. Krouwer, Akio Asai, Satoyuki Ito, \\ Takao Hoshino and Michael D. Prados
}

\begin{abstract}
Contrast enhancement on computerized tomography (CT) scans has been used in directing therapy for presumed intracranial gliomas. However, for moderately anaplastic astrocytomas (MOAAS) and highly anaplastic astrocytomas (HAAS), it provides no information about proliferative potential. The bromodeoxyuridine (BUDR) labeling index (LI), however, indicates proliferative potential, correlating with histologic malignancy and survival. An LI < $1 \%$ is a favorable indicator; $\mathrm{LI}>5 \%$ suggests more aggressiveness. To determine the correlation, if any, between BUDR LI and contrast enhancement, CT scans of 71 patients with cerebral hemisphere tumors labeled with BUDR were retrospectively reviewed. Among 36 MOAAS, the BUDR LI was $<1 \%$ in $77 \%$ of enhanced tumors and $61 \%$ of unenhanced tumors. Among 35 HAAS, it was $<5 \%$ in $56 \%$ of enhanced tumors and $90 \%$ of unenhanced tumors. Therefore, contrast enhancement on CT scans does not always correctly predict proliferative potential in these tumors, and biopsy and labeling studies are recommended before therapy.
\end{abstract}

\begin{abstract}
RÉSUMÉ: Comparaison du CT à contraste accru et indices de marquage au BUDR dans les astrocytomes modérément et hautement anaplasiques des hémisphères cérébraux. La tomodensitométrie à contraste accru a été utilisée pour diriger le traitement de gliomes intracrâniens présumés. Cependant, pour les astrocytomes modérément anaplasiques (AMAS) et les astrocytomes hautement anaplasiques (AHAS), cette technique ne fournit pas d'information au sujet du potentiel prolifératif de la tumeur. L'indice de marquage (IM) au bromodésoxyuridine (BUDR) indique le potentiel prolifératif qui est correlé avec le degré de malignité histologique et la survie. Un IM $<1 \%$ est un indicateur favorable; un IM > 5\% suggère une tumeur plus agressive. Pour déterminer la corrélation, si elle existe, entre l'IM au BUDR et l'imagerie à contraste accru, les CT scans de 71 patients avec tumeur hémisphérique cérébrale marquée au BUDR ont été revus rétrospectivement. Parmi 36 AMAS, l'IM au BUDR était $<1 \%$ dans $77 \%$ des tumeurs avec contraste accru et $61 \%$ des tumeurs sans contraste accru. Parmi 35 AHAS, il était $<5 \%$ dans 56\% des tumeurs avec contraste accru et $90 \%$ des tumeurs sans contraste accru. Donc, un rehaussement du contraste au CT scan ne prédit pas toujours avec exactitude le potentiel prolifératif de ces tumeurs et la biopsie et les études de marquage sont recommandées avant de procéder au traitement.
\end{abstract}

Can. J. Neurol. Sci. 1992; 19: 34-39

Computerized tomography (CT) scans with contrast enhancement have been used to assist in the diagnosis of patients who are thought to have brain tumors. Contrast enhancement after the intravenous administration of iodinebased dyes is often seen in tumors with abnormal and increased vascularity. Contrast enhancement can also be caused by necrosis or by the extravascular accumulation of contrast material because of an abnormal blood-brain barrier. ${ }^{1-3}$ Histopathological features such as the degree of cellularity and cellular pleomor- phism are less strongly associated with contrast enhancement than are these vascular abnormalities. ${ }^{1,4}$ Early studies showed that contrast enhancement on CT scans had 70-87\% accuracy in predicting the correct diagnosis and thus the degree of anaplasia. ${ }^{1,5-8}$ Later reviews, however, revealed that there was no exact correlation between contrast enhancement and pathology. ${ }^{4.9 .10}$

These reports also suggested that in predicting outcome for patients with astrocytomas, the presence or absence of contrast

From the Department of Neurological Surgery, Division of Neuro-Oncology (M.W.M., H.G.J.K., M.D.P.) and the Brain Tumor Research Center (A.A., S.I., T.H.), School of Medicine, University of California, San Francisco, California, U.S.A.

Received July 17, 1991. Accepted September 26, 1991

Reprint requests to: Dr. Michael D. Prados, Department of Neurological Surgery, c/o The Editorial Office, 1360 Ninth Avenue, Suite 210 , San Francisco, California, U.S.A., 94122 
enhancement would be more accurate than pathological tissue analysis, in which sampling error may occur. ${ }^{1,6-8.11}$ However, Silverman and Marks, ${ }^{12}$ in a review of 22 adults with low-grade astrocytomas who underwent operation followed by radiation therapy, found no differences in survival related to the presence or absence of contrast enhancement. In a more recent analysis of 60 patients with low-grade astrocytomas of the cerebral hemispheres, the patient's age and contrast enhancement on CT scans were the only factors with a significant influence on survival time. ${ }^{13}$ The mean survival time for 10 patients with contrastenhanced low-grade astrocytomas was 3.92 years, compared to 7.49 years for 39 patients without contrast enhancement on CT scans $(p=0.008)$. Despite these conflicting results and the lack of absolute concordance between contrast enhancement and the degree of histologic malignancy, decisions regarding treatment are still often based on radiographic characteristics, whether or not a tissue diagnosis is made. Other radiographic methods, such as magnetic resonance (MR) imaging, single photon emission computerized tomography (SPECT), magnetic resonance spectroscopy (MRS), and positron emission tomography (PET), are being used more often and may provide significantly more information than CT alone.

Hoshino and associates ${ }^{14-17}$ pioneered the use of bromodeoxyuridine (BUDR), a thymidine analog, in the analysis of the proliferative potential of malignant gliomas. The percentage of cells labeled with BUDR, or the BUDR labeling index (LI), is a measure of the proportion of cells in the S-phase of the cell cycle and thus of the tumor's proliferative potential. In a recent report of 127 patients with primary malignant gliomas, those whose tumors had LIs less than $1 \%$ had a significantly greater length of survival than did those with LIs greater than or equal to $5 \%$, irrespective of the histologic diagnosis. ${ }^{16}$ An earlier analysis of low-grade astrocytomas also showed that patients with BUDR LIs less than $1 \%$ had a significantly better 3-year survival rate than patients with higher LI values. ${ }^{17}$ Thus, the BUDR LI for supratentorial astrocytomas appears to be an accurate predictor of outcome.

At the University of California, San Francisco (UCSF), decisions about treatment of patients with low-grade astrocytomas have been influenced by the presence or absence of contrast enhancement and more recently by the BUDR LI. If contrast enhancement reflects the potential biologic behavior and proliferative capacity of a tumor, then contrast-enhanced tumors should have higher LIs than tumors in the same histopathologic group that are not enhanced with contrast. We sought to determine whether such a relationship exists between contrast enhancement and BUDR LI. We therefore conducted a retrospective review of patients with moderately anaplastic astrocytomas (MOAAS) or highly anaplastic astrocytomas (HAAS) of the cerebral hemispheres who had undergone surgery at UCSF and for whom BUDR LIs and CT scans were available.

\section{Materials AND Methods}

\section{Selection of Cases}

From among all patients who had tumors studied by biopsy after labeling with BUDR between 1984 and 1989 at UCSF, those with histologically diagnosed MOAAS or HAAS were selected (Table 1). Tumors were classified into these types by the system used at UCSF. Patients with mixed oligoastrocy- tomas were excluded from this analysis. Staining for glial fibrillary acidic protein (GFAP) was performed routinely as necessary to assess the astrocytic component of tumors. Patients with juvenile pilocytic astrocytomas were excluded because these tumors appear to have different biological characteristics from the other types; those with glioblastoma multiforme (GBM) were excluded because more than $90 \%$ of these lesions are contrast-enhanced, and patients who have them uniformly have poor survival. ${ }^{1,5,8,9,11}$ The patients for whom BUDR LIs were available included 74 with MOAAS and 68 with HAAS. Among these, patients with tumors outside the cerebral hemispheres, recurrent or mixed gliomas, or incomplete data were excluded from the study (Table 2). Therefore, 36 patients with MOAAS and 35 with HAAS were evaluated.

All patients had undergone surgery at UCSF. Tissue had been obtained from 34 (94.4\%) of the 36 evaluable patients with

Table 1: Histologic Criteria for Protoplasmic/Fibrillary Astrocytomas

Glioblastoma multiforme (GBM)

1. A glial neoplasm that, at least focally, has high cellularity

2. Nuclear pleomorphism

3. Cytoplasmic pleomorphism

4. Vascular endothelial proliferation

Highly anaplastic astrocytoma (HAA)

1. Not a glioblastoma multiforme

2. At least focally, moderate to high cellularity

3. At least two of the following:

a. high nuclear/cytoplasmic ratio

b. coarse nuclear chromatin

c. much mitotic activity

d. nuclear pleomorphism

e. cytoplasmic pleomorphism

Moderately anaplastic astrocytoma (MOAA)

1. Not a highly anaplastic astrocytoma

2. Mild/moderate increased cellularity

3. Enlarged nuclei

4. Relatively uniform cytoplasm

\begin{tabular}{lcc}
\hline Table 2: Selection of Patients for Study* & & \\
\hline \hline Category & MOAA & HAA \\
\hline Total number of patients with & & \\
available BUDR indices & 74 & 68 \\
Cases excluded: & 6 & 2 \\
$\quad$ i. location - brain stem & 2 & 2 \\
$\quad$ - cerebellum & 4 & 4 \\
$\quad$ - third ventricle & 2 & 0 \\
$\quad$ - pineal region & 1 & 0 \\
$\quad$ - spinal cord & 10 & 14 \\
$\quad 11$ & 7 \\
$\quad$ ii. recurrent glioma & 2 & 4 \\
$\quad$ iii. mixed glioma & 38 & 33 \\
$\quad$ Total exclusions & 36 & 35 \\
Number of evaluable patients &
\end{tabular}

* $\quad$ BUDR $=$ bromodeoxyuridine, MOAA = moderately anaplastic astrocytoma, $\mathrm{HAA}=$ highly anaplastic astrocytoma. 
MOAAS during craniotomy and from the other two $(5.6 \%)$ by stereotactic biopsy. Of the 35 evaluable patients with HAAS, 32 $(91.4 \%)$ had undergone craniotomy and $3(8.6 \%)$ had undergone stereotactic biopsy.

\section{BUDR Assay}

Consent for the administration of BUDR and labeling studies was obtained from each patient before operation. These labeling studies were done with the permission of the Human Experimentation Committee at UCSF.

The BUDR was administered at a dose of $200 \mathrm{mg} / \mathrm{m}^{2}$ over 30 minutes at least 1 hour before craniotomy or biopsy. Immediately after removal, tumor specimens were fixed in $70 \%$ ethanol, and after 12 hours they were embedded in paraffin. The specimens were then cut into sections $5 \mu \mathrm{m}$ thick, deparaffinized in xylene, and rehydrated in $100 \%$ ethanol followed by $95 \%$ ethanol. They were then incubated in $0.3 \%$ hydrogen peroxide for 30 minutes to block endogenous peroxidase and incubated with $4 \mathrm{~N} \mathrm{HCl}$ for 10 minutes to denature the DNA. The tissue sections were reacted with anti-BUDR monoclonal antibodies for 1 hour, incubated for 30 minutes with a perioxidaseconjugated anti-mouse immunoglobulin antibody, developed with diaminobenzidine tetrahydrochloride, and counterstained with $10 \%$ hematoxylin. Between 6 and 15 high-powered microscopic fields were examined, and at least 1000 cells in each specimen were counted. The areas selected for analysis were those that had an even distribution of labeled cells. Simultaneously obtained slides of the tumor stained with hematoxylin and eosin were used to confirm that the areas studied consisted of tumor tissue. The BUDR LI, or S-phase fraction, was calculated as the percentage of nuclei counted that were BUDR-labeled, excluding nuclei of vascular and hematogenous components.

\section{CT Scan Review}

Original CT scans were available for 18 of the 36 evaluable patients with MOAAS and for 23 of the 35 patients with HAAS. In the remaining cases, data regarding the presence or absence of contrast enhancement were taken either from the radiologist's report or from notes in the neuro-oncology chart.

CT scans were evaluated for the presence or absence of contrast enhancement but not for the pattern or degree of enhancement. Scans obtained without the use of contrast agents were used to rule out hemorrhage into the tumor. Because the scans were obtained at different institutions and the administration of contrast was not standardized, an adequate amount of contrast agent was assumed to have been given if the basal vessels were clearly visible on lower axial CT sections. In all but two cases, the presence or absence of contrast enhancement could be determined readily; the other two cases were later reviewed with a neuroradiologist, who ascertained the presence or absence of enhancement. Curvilinear enhancement at the periphery of the lesion, when near either to a superficial cortical salcus or to the sylvian fissure, was assumed to represent compressed vascular structures or venous congestion rather than enhancing tumor.

Because of the small number of cases, wide range of ages, and varying treatments, no attempt was made to analyze the data with respect to survival.

\section{RESULTS}

The male-female ratio, range of ages, and location of tumors were similar for patients with MOAAS and those with HAAS
(Table 3). The mean age was higher in the HAA group than in the MOAA group. Thirteen (36\%) of the 36 MOAAS and 25 ( $71 \%$ ) of the 35 HAAS were contrast-enhanced.

Of the 36 patients with MOAAS, $24(67 \%)$ had BUDR LIs less than $1 \%$, and only one (3\%) had an LI of $5 \%$ or more. Of the 35 patients with HAAS, $5(14 \%)$ had LIs of less than $1 \%$, and $12(34 \%)$ had LIs of $5 \%$ or more.

\section{Contrast Enhancement and LI}

\section{MOAA}

In patients with MOAAS, the contrast-enhanced tumors did not have higher LIs than tumors not enhanced with contrast. In

Table 3: Summary of Data on Moderately and Highly Anaplastic Astrocytomas*

\begin{tabular}{|c|c|c|}
\hline Characteristic & MOAA & HAA \\
\hline Number evaluable & 36 & 35 \\
\hline Male/female & $22 / 14$ & $23 / 12$ \\
\hline \multicolumn{3}{|l|}{ Age } \\
\hline $\begin{array}{l}\text { mean (years) } \\
\text { range (years) }\end{array}$ & $\begin{array}{c}29.1 \pm 18.2 \\
2-68\end{array}$ & $\begin{array}{c}40.3 \pm 20.0 \\
1-71\end{array}$ \\
\hline \multicolumn{3}{|l|}{ Location } \\
\hline frontal & 12 & 15 \\
\hline temporal & 13 & 9 \\
\hline parietal & 10 & 6 \\
\hline occipital & 0 & 1 \\
\hline other & $1^{* *}$ & $4^{* * *}$ \\
\hline \multicolumn{3}{|l|}{ Contrast enhancement } \\
\hline yes & $13(36.1 \%)$ & $25(71.4 \%)$ \\
\hline no & $23(63.9 \%)$ & $10(28.6 \%)$ \\
\hline \multicolumn{3}{|l|}{ Labeling index } \\
\hline less than $1 \%$ & $24(66.7 \%)$ & $5(14.3 \%)$ \\
\hline between $1 \%$ and $5 \%$ & $11(30.6 \%)$ & $18(51.4 \%)$ \\
\hline greater than $5 \%$ & $1(2.8 \%)$ & $12(34.3 \%)$ \\
\hline
\end{tabular}

* MOAA = moderately anaplastic astrocytoma, HAA = highly anaplastic astrocytoma.

** Caudate nucleus.

*** Two corpus callosum, 2 pulvinar.

Table 4: BUDR LI Versus Contrast Enhancement for MOAAS and HAAS*

$\begin{array}{ll}\text { 1. } & \text { Non-CE MOAA } \\ \text { LI }<1 & 60.8 \%(14 / 23) \\ \text { LI } \geq 1 & 39.2 \%(9 / 23) \\ \text { 2. } \text { CE MOAA } & \\ \text { LI }<1 & 76.9 \%(10 / 13) \\ \text { LI } \geq 1 & 23.1 \%(3 / 13) \\ \text { 3. Non-CE HAA } \\ \text { LI }<1 & 20.0 \%(2 / 10) \\ 1 \leq \mathrm{LI}<5 & 70.0 \%(7 / 10) \\ \text { LI } \geq 5 & 10.0 \%(1 / 10) \\ \text { 4. CE HAA } & \\ \text { LI }<1 & 12.0 \%(3 / 25) \\ 1 \leq \mathrm{LI}<5 & 44.0 \%(11 / 25) \\ \text { LI } \geq 5 & 44.0 \%(11 / 25)\end{array}$

* $\quad$ BUDR = bromodeoxyuridine, $\mathrm{LI}=$ labeling index, MOAA $=$ moderately anaplastic astrocytoma, $\mathrm{HAA}=$ highly anaplastic astrocytoma, $\mathrm{CE}=$ contrast-enhanced. 
this group, LIs less than $1 \%$ were found in $77 \%$ of the tumors that were contrast-enhanced and in $61 \%$ of those that were not (Table 4). Thus, for supratentorial MOAAS, contrast enhancement did not appear to reflect the proliferative potential of the tumor as measured by the BUDR LI. The MOAA with the highest LI $(8.3 \%)$ was not enhanced with contrast.

\section{HAA}

In the HAA group, the presence or absence of contrast enhancement did not indicate whether the LI was less or more than $1 \%$. The BUDR LI was $1 \%$ or more in $88 \%$ of the contrastenhanced HAAS and in $80 \%$ of the HAAS that were not enhanced with contrast. However, in the subgroup of HAAS with LIs of $1 \%$ or more, $11(50 \%)$ of the 22 contrast-enhanced HAAS and only $1(13 \%)$ of the 8 HAAS not enhanced with contrast had LIs of $5 \%$ or more. Thus, HAAS with an LI greater than or equal to $5 \%$ were more likely to show contrast enhancement than not.

\section{Discussion}

In patients with MOAAS, contrast enhancement appears to be a poor indicator of the potential biologic behavior and proliferative capacity of a tumor as measured by BUDR LI. In patients with HAAS, however, contrast enhancement is useful in identifying tumors likely to have BUDR LIs over $5 \%$.

Before the development of CT, the diagnosis of intra-axial primary malignant gliomas was often made with the assistance of cerebral angiography and radionuclide imaging studies. ${ }^{18,19}$ Although low-grade tumors are similar to the surrounding normal brain in degree of vascular supply, malignant tumors are more vascular and are supplied by abnormal vessels with irregular lumina and shapes, resulting in direct arteriovenous shunting and rapid clearance of contrast media. ${ }^{18}$ Radionuclide imaging with ${ }^{99 m}$ technetium $\left({ }^{99 m} \mathrm{Tc}\right)$ sodium pertechnetate was also often used to image the brains of patients thought to have gliomas. A review of 21 clinical series revealed that ${ }^{99 \mathrm{~m}} \mathrm{Tc}$ scanning detected the presence of $74 \%$ of grade I/II and $92.4 \%$ of grade III/IV astrocytomas. ${ }^{19}$

The development of CT scanning greatly improved the imaging of brain tumors, and CT was established as the primary investigative tool in patients who are thought to have brain tumors. The incidence, intensity, and pattern of contrast enhancement were examined, and an attempt was made to correlate these features with the histologic grade of tumor. Low-grade astrocytomas had some degree of contrast enhancement in 0$48 \%$ of cases, whereas anaplastic astrocytomas and glioblastoma multiforme showed some degree of enhancement in more than $90 \%$ of cases. ${ }^{1,2,5-11,20,21}$ In low-grade astrocytomas, the pattern of contrast enhancement was most often nodular, whereas in malignant tumors, enhancement was heterogeneous or the enhanced regions were multiloculated or ring-shaped. $2,8,10,11,21$ Butler et al. ${ }^{1}$ showed that vascularity and necrosis in supratentorial gliomas correlate with contrast enhancement. Although cellularity and pleomorphism are less closely correlated with contrast enhancement, the authors suggested that the pathologic grade of a supratentorial malignant astrocytoma can be inferred from a post-contrast CT scan. Others shared this initial enthusiasm, stating that contrast enhancement on CT scans are "more accurate a prognostic sign than pathology"7 and that the accu- racy "reached almost that of a brain slice seen at autopsy."21 However, later investigators found only a partial correlation between contrast enhancement on CT and other imaging modalities; they also found an inconsistent relationship between contrast enhancement and histologic grade. . $^{3.49}$

In a comparison of CT scans and neuropathologic findings in 8 patients, variations in the intensity of enhancement often had no apparent correlation with local differences in vascularity, cellularity, or pleomorphism. ${ }^{4}$ Therefore, no constant correlation could be drawn between contrast enhancement and malignancy. In a review of 229 patients with supratentorial malignant gliomas that were confirmed pathologically, $4 \%$ of GBM and $31 \%$ of HAAS were not enhanced with contrast agents. ${ }^{9}$ Thus, without biopsy, after the initial radiologic analysis, these tumors may have been mistaken for lower grade tumors. Histologic confirmation of the diagnosis was recommended regardless of the presence or absence of contrast enhancement.

Several histologic classification systems are now in use for grading astrocytomas, and with most there is a correlation between tumor type and survival. However, patients with a particular histologic type of tumor have a range of survival times that cannot be explained by pathology alone..$^{22}$ Piepmeier $^{13}$ has stated that the presence of contrast enhancement on CT scans indicates a worse prognosis for patients with low-grade astrocytomas. However, when this approach is used to predict survival, the results are conflicting and inconsistent. ${ }^{12}$ Contrast enhancement on CT scans is a static assessment of tumors and is related to the degree of abnormal and increased vascularity and to accumulated extravascular contrast material.

MR imaging provides greater ability to identify low-grade tumors of the deep midline and posterior fossa and is now the most useful technique for the imaging of intracranial, intra-axial tumors. ${ }^{23-25}$ In low-grade tumors, isolated tumor cells, identified by stereotactic biopsy, extend as far as the increased signal intensity on T2-weighted MR images. ${ }^{20}$ Furthermore, tumor tissue without intervening normal brain is indistinguishable on both CT scans and MR images from parenchyma infiltrated by tumor cells. In malignant gliomas, however, contrast-enhanced areas on CT accurately define the volume of tumor tissue, and increased signal intensity on $\mathrm{T} 2$-weighted images reflects the boundaries of isolated tumor cells. In another study, regions of contrast enhancement on MR images corresponded in 4 of 6 patients to neovascularity and endothelial proliferation in solid tumor tissue, and central zones of decreased contrast enhancement corresponded to areas of necrosis. ${ }^{3}$ Isolated tumor cells are seen in biopsy specimens taken from outside areas that appear abnormal on both pre-contrast and post-contrast $\mathrm{Tl}$-weighted and T2-weighted MR images. These isolated tumor cells have the biologic potential for further growth and are responsible for recurrence of tumor outside of the original site. ${ }^{26-28}$

Although contrast-enhanced CT scans and MR images reflect the physical characteristics of astrocytomas, newer imaging modalities reflect their functional and metabolic status as well. SPECT with thallium-201, MRS examining ${ }^{3 !} \mathrm{P}$ spectra, and PET using ${ }^{11} \mathrm{C}$-L-methionine and ${ }^{18} \mathrm{~F}$-2-deoxyglucose have also been used to assess the metabolic activity of tumors and to relate this to histologic grade and in some cases to survival. ${ }^{29-35}$

Another method of assessing the proliferative potential of brain tumors in vivo involves the administration of radioactive 
${ }^{3} \mathrm{H}$-thymidine and analysis of the ${ }^{3} \mathrm{H}$-thymidine $\mathrm{LI}$ of biopsy specimens. ${ }^{36-38}$ Hoshino et al. ${ }^{37}$ showed that the ${ }^{3} \mathrm{H}$-thymidine LI increases pathologic malignancy. In a later study involving 24 patients, the mean LI was $1.0 \%$ for MOAAS, $4.0 \%$ for HAAS, and $9.3 \%$ for GBM. ${ }^{38} \mathrm{~A}$ monoclonal antibody against DNA that has incorporated BUDR provides a safer method to study tumor kinetics and LI. ${ }^{39,40}$ This agent has been used in our laboratory since 1984 to study over 500 tumors in situ. Significantly higher BUDR LIs are found in gliomas with necrosis and increased vascularity than in those without these features. ${ }^{41}$ The number of mitotic nuclei per high-power field, however, does not correlate with BUDR LI.

Not only does a high BUDR LI indicate malignant pathology, but it also predicts shorter survival. In $62 \%$ of patients with lowgrade astrocytomas, the BUDR LI is less than $1 \% .{ }^{17}$ Of these patients with low BUDR LIs, $85 \%$ survive longer than 3 years; of those with BUDR LIs of $1 \%$ or more, only $10 \%$ live longer than 3 years $(p<0.01)$. Studies including patients with malignant gliomas have also shown an association between the BUDR LI, pathologic grade, and survival. Primary MOAAS, HAAS, and GBM have median BUDR LIs of less than $1 \%$, $2.7 \%$, and $7.3 \%$, respectively. ${ }^{16}$ Patients whose tumors have BUDR LIs less than $1 \%$ have the best prognosis, regardless of pathologic type, whereas those with LIs of $5 \%$ or more have the worst prognosis (median survival 40-50 weeks). Therefore, the LI of tumor tissue obtained by biopsy after the intravenous administration of BUDR offers important information about the proliferative potential of a tumor. This information may affect decisions regarding treatment and may help to predict patient outcome.

In our review, $36 \%$ of MOAAS showed some degree of contrast enhancement, and $29 \%$ of HAAS did not, confirming that there is no consistent correlation between contrast enhancement and pathologic grade. These figures are in agreement with previously published series..$^{5,9,10,12}$ In both groups, sampling error was presumably minimal, because tissue was obtained at craniotomy in over $90 \%$ of cases. Even in this select group of patients, the BUDR LI increased with increasing histologic malignancy: $33 \%$ of MOAAS and $86 \%$ of HAAS had LIs equal to $1 \%$ or more. Again, this distribution of LIs is similar to those previously reported by Hoshino et al. ${ }^{16}$ In this MOAA group, there was no relationship between contrast enhancement on CT scans the BUDR LI: $61 \%$ of the MOAAS not enhanced with contrast and $77 \%$ of the MOAAS that were contrast-enhanced had BUDR LIs less than $1 \%$. For HAAS, the presence or absence of contrast enhancement did not indicate whether the LI was more or less than 1\%; a greater percentage of the HAAS with LIs of $5 \%$ or more, however, showed contrast enhancement.

Although contrast enhancement on CT scans does not appear to accurately reflect the proliferative potential of gliomas, it may be indicative of some other inherent biologic property or properties. As newer treatment modalities are developed, and the response of tumors to these treatments is analyzed with respect to the presence or absence of contrast enhancement on CT, further definition of these properties may become possible. In the absence of this information, even functional imaging studies such as SPECT, MRS, and PET do not allow definitive prediction of the biologic behavior of individual tumors. Therefore, in patients thought to have intracranial gliomas, we recommend pathological confirmation of tumor type and estimation of the proliferative potential.

\section{ACKNOWLEDGEMENT}

This work was supported in part by National Cancer Institute grants CA 13525 and CA 50210.

\section{REFERENCES}

1. Butler AR, Horii SC, Kricheff II, et al. Computed tomography in astrocytomas. Radiology 1978; 129: 433-439.

2. Butler AR, Passalaqua AM, Berenstein A, et al. Contrast enhanced CT scan and radionuclide brain scan in supratentorial gliomas. Am J Roentgenol 1979; 132: 607-611.

3. Earnest F, Kelly PJ, Scheithauer BW, et al. Cerebral astrocytomas: histopathologic correlation of MR and CT contrast enhancement with stereotactic biopsy. Radiology 1988; 166: 823-827.

4. Lilja A, Bergstrom K, Spannare B, et al. Reliability of computed tomography in assessing histopathological features of malignant supratentorial gliomas. J Comput Assist Tomogr 1981; 5: 625636.

5. Joyce P, Bentson J, Takahashi M, et al. The accuracy of predicting histologic grades of supratentorial astrocytomas on the basis of computerized tomography and cerebral angiography. Neuroradiology 1978; 16: 346-348.

6. Kendall BE, Jakubowski J, Pullicino P, et al. Difficulties in diagnosis of supratentorial gliomas by CAT scan. J Neurol Neurosurg Psychiatry 1979; 42: 485-492.

7. Marks JE, Gado M. Serial computed tomography of primary tumors following surgery, irradiation, and chemotherapy. Radiology 1977; 125: 119-125.

8. Steinhoff H, Lanksch W, Kazner E, et al. Computed tomography in the diagnosis and differential diagnosis of glioblastomas. Neuroradiology 1977; 14: 193-200.

9. Chamberlain MC, Murovic JA, Levin VA. Absence of contrast enhancement on CT brain scans of patients with supratentorial malignant gliomas. Neurology 1988; 38: 1371-1374.

10. Tchang S, Scotti G, Terbrugge K, et al. Computerized tomography as a possible aid to histological grading of supratentorial gliomas. J Nerosurg 1977; 46: 735-739.

11. Leeds NE, Elkin CM, Zimmerman RD. Gliomas of the brain. Semin Roentgenol 1984; 19: 27-43.

12. Silverman C, Marks JE. Prognostic significance of contrast enhancement in low-grade astrocytomas of the adult cerebrum. Radiology 1981; 139: 211-213.

13. Piepmeier JM. Observations on the current treatment of low-grade astrocytic tumors of the cerebral hemispheres. J Neurosurg 1987; 67: 177-181.

14. Hoshino T. Immunohistochemical analysis of the proliferative potential of nervous system tumors. In: ISI Atlas of Science: Immunology. Philadelphia: ISI, 1988: 53-57.

15. Hoshino T, Nagashima T, Cho KG, et al. Variability in the proliferative potential of human gliomas. J Neurooncol 1989; 7: 137143.

16. Hoshino T, Prados M, Wilson CB, et al. Prognostic implications of the bromodeoxyuridine labeling index of human gliomas. $\mathrm{J}$ Neurosurg 1989; 71: 335-341.

17. Hoshino T, Rodriguez LA, Cho KG, et al. Prognostic implications of the proliferative potential of low-grade astrocytomas. J Neurosurg 1988; 69: 839-842.

18. Schecter MM. Cerebral angiography. In: Youmans JR, ed. Neurological Surgery, 2nd Ed. Vol 1. Philadelphia: WB Saunders, 1982: 231-350.

19. Wilkinson RH Jr., Goodrich JK. Radionuclide imaging studies. In: Youmans JR, ed. Neurological Surgery, 2nd Ed. Vol 1. Philadelphia: WB Saunders, 1982: 143-175.

20. Kelly PJ, Daumas-Duport C, Scheithauer BW, et al. Stereotactic histologic correlations of computed tomography- and magnetic resonance imaging-defined abnormalities in patients with glial neoplasms. Mayo Clin Proc 1987; 62: 450-459. 
21. Steinhoff H, Grumme T, Kazner E, et al. Axial transverse computed tomography in 73 glioblastomas. Acta Neurochir (Wien) 1978; 42: 45-56.

22. Morantz RA, Radiation therapy in the treatment of cerebral astrocytoma. Neurosurgery $1987 ; 20$ : 975-982.

23. Brant-Zawadzki M, Badami JP, Mills CM, et al. Primary intracranial tumor imaging: a comparison of magnetic resonance and CT. Radiology 1984; 150: 435-440.

24. Brant-Zawadzki M, Berry I, Osaki L, et al. Gd-DTPA in clinical MR of the brain. 1. Intraaxial lesions. Am J Neuroradiol 1986; 7: 781-788.

25. Kucharczyk W, Brant-Zawadzki M, Sobel D, et al. Central nervous system tumors in children: detection by magnetic resonance imaging. Radiology 1985; 155: 131-136.

26. Bashir R, Hochberg F, Oot R. Regrowth patterns of glioblastoma multiforme related to planning of interstitial brachytherapy radiation fields. Neurosurgery 1988; 23: 27-30.

27. Hochberg FH, Pruitt A. Assumptions in the radiotherapy of glioblastoma. Neurology 1980; 30: 907-911.

28. Wallner KE, Galicich JH, Krol G, et al. Patterns of failure following treatment for glioblastoma multiforme and anaplastic astrocytoma. Int J Radiat Oncol Biol Phys 1989; 16: 1405-1409.

29. Black KL, Hawkins RA, Kim KT, et al. Use of thallium-201 SPECT to quantitate malignancy grade of gliomas. J Neurosurg 1989; $71: 342-346$.

30. Derlon JM, Bourdet C, Bustany P, et al. $\left({ }^{11} \mathrm{C}\right) \mathrm{L}$-methionine uptake in gliomas. Neurosurgery $1989 ; 25: 720-728$.

31. Kaplan WD, Takvorian T, Morris JH, et al. Thallium-201 brain tumor imaging: a comparative study with pathologic correlation. J Nucl Med 1987; 28: 47-52.
32. Lilja A, Lundquist H, Olsson $\mathrm{Y}$, et al. Positron emission tomography and computed tomography in differential diagnosis between recurrent or residual glioma and the treatment-induced brain lesions. Acta Radiol 1989; 30: 121-128.

33. Mountz JM, Stafford-Schuck K, McKeever PE, et al. Thallium-201 tumor/cardiac ratio estimation of residual astrocytoma. J Neurosurg 1988; 68: 705-709.

34. Patronas NJ, Di Chio G, Kufta C, et al. Prediction of survival in glioma patients by means of positron emission tomography. $\mathrm{J}$ Neurosurg 1985; 62: 816-822.

35. Sutton LN, Lenkinski RE, Cohen $\mathrm{BH}$, et al. Localized ${ }^{31} \mathrm{P}$ magnetic resonance spectroscopy of large pediatric brain tumors. J Neurosurg 1990; 72: 65-70.

36. Hoshino T. A commentary on the biology and growth kinetics of low-grade and high-grade gliomas. J Neurosurg 1984; 61: 895900.

37. Hoshino T, Barker M. Wilson CB, et al. Cell kinetics of human gliomas. J Neurosurg 1972; 37: 15-26.

38. Hoshino T, Wilson CB. Cell kinetic analysis of human malignant brain tumors (gliomas). Cancer 1979; 44: 956-963.

39. Gratzner HG. Monoclonal antibody to 5-bromo- and 5-iododeoxyuridine: a new reagent for detection of DNA replication. Science 1982; 218: 474-476.

40. Nagashima T, DeArmond SJ, Murovic J, et al. Immunocytochemical demonstration of S-phase cells by anti-bromodeoxyuridine monoclonal antibody in human brain tumor tissues. Acta Neuropathol (Berl) 1985; 67: 155-159.

41. Germano IM, Ito M, Cho KG, et al. Correlation of histopathological features and proliferative potential of gliomas. J Neurosurg 1989; 70: 701-706. 